\title{
Association Between Blood Pressure and
}

\section{Branched-Chain/Aromatic Amino Acid Excretion Rate in 24-Hour Urine Samples from Elderly Hypertension Patients}

\author{
Faxuan Wang $\mathbb{D}^{1,2, *}$ \\ Binxia Wang ${ }^{3, *}$ \\ Xiyuan Chen ${ }^{1,2}$ \\ Wanlu Liu ${ }^{1,2}$ \\ Guoqi Wang ${ }^{1,2}$ \\ Xiaoxia $\mathrm{Li}^{1,2}$ \\ Xiuying Liu ${ }^{1,2}$ \\ Nan $\mathrm{Li}^{1,2}$ \\ Jiaxing Zhang ${ }^{1,2}$ \\ Ting Yin ${ }^{1,2}$ \\ Jinyun Jing ${ }^{1,2}$ \\ Xiaoyu Chang ${ }^{4}$ \\ Yanan Jin ${ }^{5}$ \\ Yuhong Zhang ${ }^{1,2}$ \\ Yi Zhao (D)'
}

'School of Public Health and Management, Ningxia Medical University, Yinchuan,

Ningxia, People's Republic of China; ${ }^{2}$ Key Laboratory of Environmental Factors and

Chronic Disease Control, Yinchuan,

Ningxia, People's Republic of China;

${ }^{3}$ Second People's Hospital of Gansu

Province, Lanzhou, Gansu, People's

Republic of China; ${ }^{4}$ Editorial Department of the Journal of Ningxia Medical University,

Ningxia Medical University, Yinchuan,

Ningxia, People's Republic of China;

${ }^{5}$ Centers for Disease Control and

Prevention in Ningxia, Yinchuan, Ningxia,

People's Republic of China

*These authors contributed equally to this work

Correspondence: Yi Zhao

School of Public Health and Management,

Ningxia Medical University

Tel +86 I39 950। I690

Fax $+86951-6980144$

Email zhaoyi75III4@hotmail.com
Background: Recently, the association between lifestyle-related diseases and free amino acids in the blood plasma - especially branched-chain amino acids (BCAAs) and aromatic amino acids (AAAs) - has been highlighted. However, few studies have been carried out on 24-hour urine samples. Therefore, we aimed to explore the relationships between 24-hour urinary BCAAs and AAAs excretion rate and blood pressure (BP) in elderly patients with hypertension.

Methods and Results: Each of the 322 elderly patients with hypertension completed an inperson questionnaire interview, underwent a physical examination, and provided a 24-hour urine specimen. We measured their BCAAs and AAAs excretion rate, and used multiple linear regression analysis with variable selection to construct models describing the relationships between their BCAAs and AAAs excretion rate and BP. After adjusted for age, gender, height, and weight, valine was inversely associated with both systolic blood pressure (SBP) $(\beta$ : $-0.232,95 \% \mathrm{CI}:-0.16,-0.006)$ and diastolic blood pressure (DBP) $(\beta$ : $-0.144,95 \% \mathrm{CI}$ : $-0.089,-0.005)$. These findings were invariant even following adjustment for urine volume and drugs history, and Ile was positively associated with DBP $(\beta: 0.170,95 \%$ CI: 0.001 , 0.066).

Conclusion: The data revealed that the excretion rate of 24-hour urinary BCAAs was closely related to BP in elderly hypertension patients, and these findings will provide new insights into the association between BACC metabolism and BP.

Keywords: hypertension, branched-chain amino acid, aromatic amino acid, association

\section{Introduction}

Hypertension is one of the leading causes of cardiovascular disease, and the major risk factor for death around the world. ${ }^{1}$ Among Chinese adults aged 35-75 years, the age-standardized and gender-standardized prevalence of hypertension is $37.2 \%$, and the prevalence is as high as $58.4 \%$ among people aged over 60 years. ${ }^{2}$ Therefore, it is essential to study people aged 60 years and older with hypertension to further prevent cardiovascular disease.

Genetics, age, obesity, smoking, alcohol consumption, lack of exercise, and an unhealthy diet have been recognized as the major risk factors for hypertension, and ${ }^{3}$ multilayered omics studies have found that hypertension can cause renal injury, and metabolic or dietary intervention can prevent and treat glomerular disease and 
hypertensive nephropathy. ${ }^{4}$ Among the dietary factors, a meta-analysis of 40 randomized, controlled trials indicates that both animal and vegetable protein intake can markedly lower blood pressure (BP). ${ }^{5}$ It has been speculated that the antihypertensive effect of dietary proteins depends on the sources of the protein and its amino acid content, although it is completely unclear how protein intake affects BP. ${ }^{6,7}$ Former studies have suggested that glutamic acid, ${ }^{8}$ glycine, ${ }^{9}$ and L-arginine ${ }^{10}$ intake can lower BP; and methionine, alanine, ${ }^{7}$ lysine and cysteine ${ }^{11}$ intake are associated with elevated BP. Branched-chain and aromatic amino acids (BCAAs and AAAs, respectively) are important biomarkers that are significantly and positively associated with the risk factors for coronary artery disease (CAD), for instance body mass index (BMI), waist circumference, BP, and fasting blood glucose levels. ${ }^{12}$ In fact, there is growing evidence that plasma BCAA and AAA levels can be used to assess the risks of hypertension adjusted for age and gender, ${ }^{13}$ and that higher intake of such amino acids is clearly related to lower systolic BP (SBP). ${ }^{6,14}$ And the previous research found that mean 24hour urinary excretion of alanine and hippurate are also associated with blood pressure of individuals. ${ }^{15}$ However, there have been few studies analyzing BCAA and AAA levels in 24-hour urine samples in relation to BP among patients with hypertension. As the final product of metabolism, urine is more easily obtained than plasma, and can reflect the metabolic condition of the body; furthermore, 24-hour urinary metabolite concentrations are not affected by sampling time or diurnal variation, which makes urine ideal for metabolic studies. ${ }^{16-18}$ Therefore, the main objectives of the present study were to assess the association between 24-hour urinary BCAA, AAA concentration, and $\mathrm{BP}$ in elderly hypertension patients, and to further provide a theoretical basis for regulating BP.

\section{Methods}

\section{Study Population}

Our study was ancillary study of a large-scale cluster randomized controlled trial, the Salt Substitute and Stroke Study (SSaSS). ${ }^{19}$ A total of 120 villages were conducted in the Pingluo County and Qingtongxia County, of Ningxia Hui Autonomous Region, China. Approximately thirty-five high-risk stroke individuals were recruited from each village. The inclusion criteria including: 1) a history of stroke; 2) or at least 60 years old, and had uncontrolled high $\mathrm{BP}$ (SBP $\geq 140 \mathrm{mmHg}$ at visit if taking antihypertensive drugs; SBP $\geq 160 \mathrm{mmHg}$ if not taking antihypertensive drugs). The project village was randomly selected in the course of follow-up. In each village, a list of twenty subjects was generated, and the people were interviewed. If anyone of the initial list was unable to participate in the study, the replacement was chosen from the remaining population in a random number size order.

This study was based on follow-up visits in the year of 2016 and 2017. The patients were excluded from our study if they: 1) had a history of any type of cardiovascular and cerebrovascular diseases; 2) had a history of other known chronic metabolic disorders including diabetes, severe infections, autoimmune diseases, acute and chronic kidney diseases and cancer; 3) had a history of alcohol or substance abuse; 4) did not provide informed consent. We further excluded people with missing data on questionnaire, anthropometric measuring and experiment, and finally, 322 participants were included in the analysis. The Ethics Committee of Ningxia Medical University approved the present study (No. 2013-175). All participants were informed about the purpose of the study, and that it was conducted in accordance with the Declaration of Helsinki, then all patients gave their consent for participation in writing, or if that was not possible, consent was provided by appropriate family members.

\section{Questionnaire and Anthropometric Measurements}

All participants completed an interviewer-administered survey. The questionnaire included anthropometric-related information, such as age, gender, education, smoking history (defined as smoking at least one cigarette per day and smoking continuously $\geq 1$ year), current smoking habit (defined as having a history of smoking and, in the last 30 days, the average daily smoking $\geq 1$ ), chronic disease status (including transient ischemic attack, ischemic heart disease, congestive heart failure, peripheral arterial disease, hypertension, and diabetes mellitus), and drugs use in the previous 3 months (see Drugs history of Table 1 for details). Then, standing height was measured using a portable ruler and recorded to the nearest $1 \mathrm{~cm}$, and, weight was also measured using a weight scale (Omron, China) and recorded to the nearest $0.1 \mathrm{~kg}$. All measurements were performed by trained and qualified investigators, and the subjects were dressed in light clothing without wearing hats and shoes. 
Table I Demographic Characteristics of the Study Subjects According to Quartiles of Total BCAAs

\begin{tabular}{|c|c|c|c|c|c|}
\hline Characteristics & $Q_{1}(81)$ & $Q_{2}(80)$ & $Q_{3}(82)$ & $Q_{4}(79)$ & P-value \\
\hline Age (year)* & $70(67,76)$ & $68(65,75)$ & $68(65,72)$ & $67(64,71)$ & 0.001 \\
\hline Height (cm) & $157.3 \pm 8.5$ & $158.5 \pm 8.1$ & $160.0 \pm 7.7$ & $162.7 \pm 8.8$ & $<0.001$ \\
\hline Weight (kg) & $62.85 \pm 10.3$ & $63.4 \pm 11.5$ & $65.6 \pm 10.4$ & $70.0 \pm 11.4$ & $<0.001$ \\
\hline Urine volume $(\mathrm{mL})^{*}$ & $800(625,1050)$ & $950(662,1400)$ & $1194(800,1620)$ & $1400(1000,1900)$ & $<0.001$ \\
\hline Gender (female) & 46 (56.8\%) & 50 (62.5\%) & $34(41.5 \%)$ & $30(38.0 \%)$ & 0.004 \\
\hline Smoking history & $16(19.8 \%)$ & 17 (2I.3\%) & $24(29.3 \%)$ & $22(27.8 \%)$ & 0.403 \\
\hline Current smoking habit & 7 (8.6\%) & $8(10.0 \%)$ & $13(15.9 \%)$ & II (I3.9\%) & 0.462 \\
\hline Education (primary) & 79 (97.5\%) & 78 (97.5\%) & 75 (91.5\%) & 72 (91.1\%) & 0.101 \\
\hline \multicolumn{6}{|l|}{ Drugs History } \\
\hline Diuretics & 14 (I7.3\%) & $12(\mid 5.0 \%)$ & $14(17.1 \%)$ & $9(11.4 \%)$ & 0.709 \\
\hline ACEI & $26(32.1 \%)$ & $29(36.3 \%)$ & $30(36.6 \%)$ & $31(39.2 \%)$ & 0.823 \\
\hline$\alpha$-receptor blockers & $5(6.2 \%)$ & $2(2.5 \%)$ & $3(3.7 \%)$ & $4(5.1 \%)$ & 0.673 \\
\hline$\beta$-receptor blockers & 7 (8.6\%) & 9 (II.3\%) & $10(12.2 \%)$ & $13(16.5 \%)$ & 0.499 \\
\hline Calcium antagonists & $42(51.9 \%)$ & $45(56.3 \%)$ & $50(61.0 \%)$ & $52(65.8 \%)$ & 0.309 \\
\hline Other antihypertensives & $16(19.8 \%)$ & II (I3.8\%) & II (I3.4\%) & $9(\mathrm{II} .4 \%)$ & 0.472 \\
\hline Lipid-lowering drugs & $7(8.6 \%)$ & $3(3.8 \%)$ & $4(4.9 \%)$ & $3(3.8 \%)$ & $0.46 \mathrm{I}$ \\
\hline Antiplatelet agents & $24(29.6 \%)$ & $20(25.0 \%)$ & $22(26.8 \%)$ & $18(22.8 \%)$ & 0.790 \\
\hline
\end{tabular}

Note: * Values shown are Median (P25, P75).

Abbreviations: $A C E I$, angiotensin conversion enzyme inhibitors; total BCAAs, valine + isoleucine + leucine; $Q_{1}$, first quartile; $Q_{2}$, second quartile; $Q_{3}$, third quartile; $\mathrm{Q}_{4}$, fourth quartile.

\section{BP Measurement}

We used an electronic sphygmomanometer (Omron-HEM 7301-IT, China) to measure the sitting position BP at the end of the physical examination. The procedure was as follows: Each subject rested for 15 minutes, avoiding exercise, smoking, drinking, drinking tea or coffee; and if the subjects needed to empty the bladder, they should be asked to empty before taking the measurement. The right upper arm of the seat was taken in the measurement, and each subject was required to measure two times and the time interval was at least 5 minutes. If the difference between the two SBP was greater than $10 \mathrm{mmHg}$, a third measurement was needed, then recorded the last two readings, which was accurate to $1 \mathrm{mmHg}$. The average of the two readings was calculated as the subjects' BP.

\section{Collection and BCAAs/AAAs Excretion Rate Measurement of the 24-Hour Urine Samples}

We collected the 24-hour urine samples immediately after the questionnaire and stored them at $-80^{\circ} \mathrm{C}$ until required. The collection of 24-hour urine samples has previously been described in greater detail. ${ }^{20}$ All urine samples were processed, and the processed procedure was as follows: First, we took out the frozen preserved urine to thaw at room temperature, and took $1.0 \mathrm{~mL}$ urine sample to $5.0 \mathrm{~mL}$ volumetric flask. Next, in order to get deproteinized, we added 10\% sulfosalicylic acid (Tianjin damao chemical reagent factory, China) to the urine sample up to $5.0 \mathrm{~mL}$. Then, we used $10 \mathrm{~mol} / \mathrm{L}$ of sodium hydroxide (Sinopharm chemical reagent co. LTD, China) to adjust the PH value to about 2.2 and centrifugated $10 \mathrm{~min}$ at $3000 \mathrm{r} / \mathrm{min}$ (Shanghai anting, China). Finally, we took the liquid supernatant $1.5 \mathrm{~mL}$ and filtered it into the sample bottle with a 0.45 $\boldsymbol{\mu} \mathbf{m}$ microfiltration membrane (PALL, America) and placed it in $4^{\circ} \mathrm{C}$ refrigerator for testing. We used an L-8900 Automatic Amino Acid Extraction Analyzer (Hitachi, Japan) to determine the BCAAs and AAAs excretion rate, then qualitatively obtains various amino acids by retention time and quantitatively obtains peak area compared with amino acid standard maps, and finally calculates the excretion rate of amino acids by a single point external standard method $(\mathrm{Xi}=\mathrm{Ai} * \mathrm{Xs} / \mathrm{As}, \mathrm{Xi}$ and Ai represent the quality of the components contained in the sample volume and the corresponding peak area, respectively; Xs and As represent the quality of the components contained in the sample volume of the standard solution and the corresponding peak area, respectively). The Hitachi L-8900 system was operated according to the following parameters: Analytical column: $4.6 \times 60 \mathrm{~mm}$ ion-exchange resin filled column; 
reaction column: $4.6 \times 40 \mathrm{~mm}$ strongly acidic $\mathrm{Na}$ cation exchange resin-filled column; pump 1 pressure: $<10 \mathrm{MPa}$, flow rate: $0.400 \mathrm{~mL} / \mathrm{min}$; pump 2 pressure: $<2 \mathrm{MPa}$, flow rate: $0.350 \mathrm{~mL} / \mathrm{min}$; detection time: $30 \mathrm{~min}$ per patch; column oven: $57^{\circ} \mathrm{C}$; reactor heater: $135^{\circ} \mathrm{C}$; sample volume: $20.0 \mu \mathrm{L}$.

\section{Statistical Analysis}

All statistical analyses were carried out using SPSS Statistics 23.0, and statistical significance was defined as a two-sided $P$-value of less than 0.05 . Subjects were divided into four equal groups according to quartiles of total BCAAs or AAAs excretion rate. At different amino acid excretion, all continuous variables were verified for normal distribution of the data (Normality evaluation needs to be done by normality plots with tests, and the specific steps are as follows: Explore is performed in the Analyze module in SPSS23.0 software), and were expressed as mean values \pm standard deviation in for normally distributed data, or medians (interquartile ranges) for non-normally distributed; group differences were evaluated using ANOVA or Kruskal-Wallis $H$-test for non-normally distributed data. Categorical variables were described as frequencies (percentages), and Chisquare or Fisher's exact test were used to compare differences among groups. We assessed the independent association of the measured variables with BP using generalized linear regression models, and used collinearity testing to avoid the inclusion of interdependent model variables. Additional adjustments were made, including age, gender, height, weight, urine volume, missing volume and drugs history. Valine (Val) and isoleucine (Ile) were separately stratified into quartiles, and means and 95\% confidence intervals (95\% CI) of BP were compared independently using LSD. Finally, linear trend tests were implemented to show changes in BP with the BCAAs excretion rate.

\section{Results}

\section{Characteristics of the Patients Enrolled in the Study}

In total, 322 eligible participants were included in the analysis. Approximately, half of the participants were female (n: $160,49.7 \%$ ). In a preliminary analysis, the subjects were divided into quartiles according to total BCAAs or AAAs excretion rate $(\mathrm{mg} / \mathrm{d})$. The quartile cut-offs were as follows: 1.4800, 2.1850, and 3.1400 for BCAAs; and 13.8950, 23.6900, and 38.9825 for AAAs. The number of subjects and their demographic characteristics have been summarized in Tables 1 and 2. The average age of the study population was 68 , and those in the top quartiles of the BCAAs excretion rate were younger than those in the lowest

Table 2 Demographic Characteristics of the Study Subjects According to Quartiles of Total AAAs

\begin{tabular}{|c|c|c|c|c|c|}
\hline Characteristics & $Q_{1}(80)$ & $Q_{2}(81)$ & $Q_{3}(8 I)$ & $Q_{4}(80)$ & P-value \\
\hline Age (year)* & $70(66,76)$ & $69(66,74)$ & $67(64,73)$ & $67(64,7 I)$ & 0.013 \\
\hline Height (cm) & $157.3 \pm 8.0$ & $157.5 \pm 7.7$ & $160.4 \pm 7.9$ & $163.2 \pm 9.0$ & $<0.001$ \\
\hline Weight (kg) & $62.6 \pm 10.5$ & $62.8 \pm 10.4$ & $64.8 \pm 11.0$ & $70.4 \pm 11.6$ & $<0.001$ \\
\hline Urine volume* & $925(700,1200)$ & $900(700,1500)$ & $1150(690,1550)$ & $1355(1000,1750)$ & $<0.001$ \\
\hline Gender (female) & $5 \mathrm{I}(63.8 \%)$ & 45 (55.6\%) & 37 (45.7\%) & 27 (33.8\%) & 0.001 \\
\hline Smoking history & 15 (I8.8\%) & $17(21.0 \%)$ & $26(32.1 \%)$ & $21(26.3 \%)$ & 0.201 \\
\hline Current smoking habit & $6(7.5 \%)$ & $9(11.1 \%)$ & $12(14.8 \%)$ & $12(15.0 \%)$ & 0.414 \\
\hline Education (primary) & 78 (97.5\%) & 79 (97.5\%) & 76 (93.8\%) & 71 (88.8\%) & 0.057 \\
\hline \multicolumn{6}{|l|}{ Drugs history } \\
\hline Diuretics & 19 (23.8\%) & $10(12.3 \%)$ & 9 (II.1\%) & II (I3.8\%) & 0.101 \\
\hline ACEI & 25 (31.3\%) & 30 (37.0\%) & 29 (35.8\%) & 32 (40.0\%) & 0.711 \\
\hline$\alpha$-receptor blockers & $5(6.3 \%)$ & $3(3.7 \%)$ & $2(2.5 \%)$ & $4(5.0 \%)$ & 0.662 \\
\hline$\beta$-receptor blockers & $9(11.3 \%)$ & $6(7.4 \%)$ & 15 (I8.5\%) & $9(11.3 \%)$ & 0.178 \\
\hline Calcium antagonists & $4 \mathrm{I}(5 \mathrm{I} .3 \%)$ & 50 (61.7\%) & 47 (58.0\%) & 51 (63.8\%) & 0.392 \\
\hline Other antihypertensives & 17 (21.3\%) & II (I3.6\%) & II (I3.6\%) & $8(10.0 \%)$ & 0.228 \\
\hline Lipid-lowering drugs & $6(7.5 \%)$ & $4(4.9 \%)$ & $3(3.7 \%)$ & $4(5.0 \%)$ & 0.758 \\
\hline Antiplatelet agents & $23(38.8 \%)$ & $23(28.4 \%)$ & $14(17.3 \%)$ & $24(30.0 \%)$ & 0.221 \\
\hline
\end{tabular}

Note: *Values shown are Median (P25, P75).

Abbreviations: $A C E I$, angiotensin conversion enzyme inhibitors; total AAAs, tyrosine + phenylalanine + tryptophan; $Q_{1}$, first quartile; $Q_{2}$, second quartile; $Q_{3}$, third quartile; $Q_{4}$, fourth quartile. 
groups; however, height, weight and urine volume were higher in the top quartiles than the lowest quartile. There were no significant differences in smoking history, current smoking habit, education and drugs history across the BCAAs quartiles (P-value $>0.05$ ). Interestingly, the characteristics revealed similar pattern when the population was grouped by AAAs excretion rate (Table 2).

\section{Changes in Blood Pressure at Various Amino Acid Levels}

To evaluate the BP levels of subjects at BCAAs or AAAs excretion rate, we classified the population according to their BCAAs and AAAs quartiles. In general, there seemed to be no difference in blood pressure between the different quartiles of BCAAs or AAAs excretion rate in the study population (Table 3 ).

\section{Multiple Linear Regression Model}

To evaluate the multivariate correlations, we subjected all the variables listed in Table 3 to a multiple linear regression analysis as independent variables. This allowed us to identify crucial contributors to the distribution of BP. Hierarchical regression analysis revealed a similar pattern for 24-hour urine BCAAs, AAAs and BP. As exhibited in Table 4, after adjusting for age, gender, height, and weight, multiple analyses demonstrated that Val was inversely associated with both SBP and DBP $(\beta$ : $-0.232,95 \%$ CI: $-0.116 \sim-0.006$; $\beta$ : $-0.149,95 \% \mathrm{CI}:-0.089 \sim-0.005$, respectively). To test the robustness of our major findings, we conducted two adjusted analyses. After adjustment for urine volume, drugs history, the results between the Val and BP were not influenced to any appreciable extent, and Ile was positively associated with DBP $(\beta: 0.170,95 \%$ CI: $0.001,0.066)$. Notably, the majority of these analyses did not reveal any correlation between the AAAs and BP.

The multivariable models revealed significant differences in the correlations between the BCAAs excretion rate and the SBP and DBP. We created error bars to further quantify the associations between the BCAAs and BP in the present study. None of the P-value for the ANOVA indicated statistical significance, but compared to the lowest, there was a downward trend for SBP and DBP at the highest Val; and there was an upward trend for DBP at the highest Ile (Figure 1).
Table 3 Average SBP and DBP in Quartiles of BCAAs and AAAs in 24-Hour Urine Samples

\begin{tabular}{|c|c|c|c|}
\hline & & SBP & DBP \\
\hline \multirow[t]{5}{*}{ Val } & $Q_{1}$ & $162.8 \pm 22.7$ & $93.0 \pm 14.7$ \\
\hline & $\mathrm{Q}_{2}$ & $162.8 \pm 20.2$ & $93.3 \pm 13.4$ \\
\hline & $\mathrm{Q}_{3}$ & $163.1 \pm 20.1$ & $93.5 \pm 13.7$ \\
\hline & $\mathrm{Q}_{4}$ & $161.7 \pm 21.7$ & $92.2 \pm 15.0$ \\
\hline & P-value & 0.975 & 0.944 \\
\hline \multirow[t]{5}{*}{ Ile } & $Q_{1}$ & $163.8 \pm 23.2$ & $91.4 \pm 13.4$ \\
\hline & $\mathrm{Q}_{2}$ & $163.0 \pm 21.7$ & $94.8 \pm 14.9$ \\
\hline & $\mathrm{Q}_{3}$ & $161.6 \pm 18.6$ & $93.2 \pm 14.5$ \\
\hline & $\mathrm{Q}_{4}$ & $162.0 \pm 20.8$ & $92.6 \pm 13.7$ \\
\hline & P-value & 0.908 & 0.487 \\
\hline \multirow[t]{5}{*}{ Leu } & $Q_{1}$ & $163.0 \pm 24.8$ & $92.7 \pm 14.0$ \\
\hline & $\mathrm{Q}_{2}$ & $160.3 \pm 16.5$ & $91.8 \pm 13.2$ \\
\hline & $\mathrm{Q}_{3}$ & $163.8 \pm 21.2$ & $93.2 \pm 14.5$ \\
\hline & $\mathrm{Q}_{4}$ & $163.3 \pm 21.3$ & $94.4 \pm 15.0$ \\
\hline & P-value & 0.721 & 0.715 \\
\hline \multirow[t]{5}{*}{ Tyr } & $Q_{1}$ & $161.5 \pm 23.6$ & $91.3 \pm 13.6$ \\
\hline & $\mathrm{Q}_{2}$ & $163.6 \pm 18.1$ & $94.6 \pm 13.0$ \\
\hline & $\mathrm{Q}_{3}$ & $163.6 \pm 21.1$ & $92.9 \pm 15.3$ \\
\hline & $\mathrm{Q}_{4}$ & $161.7 \pm 21.7$ & $93.2 \pm 14.7$ \\
\hline & P-value & 0.876 & 0.526 \\
\hline \multirow[t]{5}{*}{ Trp } & $Q_{1}$ & $162.5 \pm 23.4$ & $91.5 \pm 14.1$ \\
\hline & $\mathrm{Q}_{2}$ & $163.0 \pm 18.8$ & $93.9 \pm 13.3$ \\
\hline & $\mathrm{Q}_{3}$ & $160.1 \pm 19.5$ & $91.6 \pm 13.3$ \\
\hline & $\mathrm{Q}_{4}$ & $164.9 \pm 22.5$ & $95.0 \pm 15.6$ \\
\hline & P-value & 0.547 & 0.292 \\
\hline \multirow[t]{5}{*}{ Phe } & $Q_{1}$ & $162.7 \pm 20.3$ & $93.8 \pm 13.0$ \\
\hline & $\mathrm{Q}_{2}$ & $165.2 \pm 25.2$ & $94.6 \pm 15.8$ \\
\hline & $\mathrm{Q}_{3}$ & $163.2 \pm 19.3$ & $91.5 \pm 14.1$ \\
\hline & $\mathrm{Q}_{4}$ & $159.4 \pm 18.6$ & $92.2 \pm 13.6$ \\
\hline & P-value & $0.38 I$ & 0.482 \\
\hline
\end{tabular}

Abbreviations: Val, valine; lle, isoleucine; Leu, leucine; Tyr, tyrosine; Phe, phenylalanine; Trp, tryptophan; $Q_{1}$, first quartile; $Q_{2}$, second quartile; $Q_{3}$, third quartile; $\mathrm{Q}_{4}$, fourth quartile.

\section{Discussion}

In this population-based, cross-sectional study, we examined the relationships between 24-hour urinary BCAA and AAA levels and BP in 322 elderly hypertension patients after adjusting for multiple confounders. Our main findings were as follows: (i) Val was inversely associated with SBP and DBP; and (ii) Ile was positively associated with DBP.

Amino acids are the basic units that make up proteins, and normal growth and maintenance of health in humans requires all amino acids to be provided in appropriate quantity. BCAAs and AAAs consist of five essential 
Table 4 Multivariable-Adjusted $\beta$ and $95 \% \mathrm{Cl}$ Value of Amino Acids and BP

\begin{tabular}{|l|l|l|l|l|l|l|}
\hline & \multicolumn{2}{|c|}{ Model I } & \multicolumn{2}{c|}{ Model 2 } & \multicolumn{2}{c|}{ Model 3 } \\
\hline & Variables & \multicolumn{1}{|c|}{$\boldsymbol{\beta}(\mathbf{9 5} \% \mathbf{C l})$} & Variables & $\boldsymbol{\beta} \mathbf{( 9 5 \% ~ C l )}$ & Variables & $\boldsymbol{\beta}(\mathbf{9 5} \% \mathbf{C l})$ \\
\hline SBP & Val & $-0.232(-0.116,-0.006)$ & Val & $-0.325(-0.147,-0.024)$ & Val & $-0.301(-0.140,-0.017)$ \\
DBP & Val & $-0.144(-0.089,-0.005)$ & Val & $-0.194(-0.113,-0.015)$ & Val & $-0.328(-0.171,-0.044)$ \\
& & & & & lle & $0.170(0.001,0.066)$ \\
\hline
\end{tabular}

Notes: Model I: adjusted for age, gender, height, weight; Model 2: Model I + urine volume; Model 3: Model $2+$ drugs history; BP and amino acids were converted by log. Abbreviations: $\beta$, the standardized partial regression coefficient; $95 \% \mathrm{Cl}, 95 \%$ confidence interval; Collinearity testing was used to avoid inclusion of interdependent model variables.

amino acids - (Val, Leu, Ile, Phe, and tryptophan (Trp)) and a conditionally essential amino acid (tyrosine (Tyr)). They play vital roles in such processes as protein degradation and turnover, the synthesis of specific neurotransmitters, and energy metabolism. ${ }^{21,22}$ The previous study showed that age and diabetes may influence plasma BCAAs, cystine and glutamine levels, ${ }^{23}$ and BCAAs, and AAAs of young adults differ by gender and body mass index, ${ }^{24,25}$ which were consistent with the results of our study. The finding of above results is of particular clinical interest and might suggest the need for further studies on the mechanisms of BCAAs and AAAs in elderly hypertension patients.

A number of researchers have reported that the serum levels of BCAAs and AAAs are significantly different among patients with metabolic syndrome, diabetes, dyslipidemia, and hypertension ${ }^{13,26}$ and are early indicators of cardiovascular disorders, ${ }^{12,27}$ and the reduction of plasma BCAA levels plays a vital role in the prevention and management of hypertension. ${ }^{28}$ Recently, the significant associations between the six amino acids and BP and future hypertension have been surveyed. However, the relationships between BCAAs and AAAs and BP varied owing to the selection of the study subjects. The relationship between the levels of circulating plasma BCAAs and the incidence of CAD has been verified in a prospective cohort of US women. ${ }^{29}$ BCAAs - especially Val and Leu-have been proposed as a cardiometabolic risk marker that is independent of BMI category. ${ }^{30} \mathrm{~A}$ former study has shown that high levels of BCAAs are beneficial for people with type 2 diabetes, and even that a high intake of BCAAs has a favorable effect on the condition. ${ }^{31}$ However, the results from existing research suggest that such relationships are completely inconsistent. Several investigators have suggested that the levels of Val are positively correlated with SBP and DBP, ${ }^{12,13,32}$ whereas in the present study Val was negatively correlated with BP. In other words, Val may have potential protective effect on BP. This potential mechanism can appear as a protein that may be associated with ion channels, which can indirectly affect the pathways of BP control. Another possible explanation is that proteins may induce a slight reduction in the levels of insulin and glucose, and hyperinsulinemic status is directly correlated to the pathophysiology of hypertension. ${ }^{33}$ Furthermore, in a crosssectional study of 1898 female twins, higher intakes of 7 potentially cardioprotective amino acids (arginine, cysteine, glutamic acid, glycine, histidine, Leu, and Tyr) are associated with lower central SBP. ${ }^{6}$ However, Leu was not correlated with SBP in the present study, and our results were also inconsistent with previous findings. ${ }^{30}$ Moreover, Ile levels are considered to be positively related to DBP in juveniles $^{30}$ and those undergoing physical examinations, ${ }^{12}$ and these findings are in agreement with our data, even the inverse correlation apparent in severely frail patients. ${ }^{34}$ Therefore, the relationship between BCAAs and BP should be further explored and verified in a large population.

We found no significant relationship between AAAs and BP in the present study. The findings regarding AAAs are controversial based on different study designs. For example, a number of researchers have reported that a greater intake of Phe is associated with an increased risk of hypertension, and can elevate BP. ${ }^{7,35,36}$ However, in severely frail elderly patients, plasma Phe is negatively associated with $\mathrm{DBP},{ }^{34}$ and Wang et al reported that serum Phe is not related to BP in 600 Chinese individuals. ${ }^{32}$

Our study has several prominent strengths. To the best of our knowledge, compared to other cross-sectional studies on the association between amino acids and BP, the present study is the first cross-sectional investigation of the relationship between 24-hour urinary BCAAs and AAAs excretion rate and BP in elderly hypertension patients. Our findings highlight the need to verify the role of BCAAs and AAAs in BP because the available literature is deficient in this regard. Furthermore, the metabolite profiling at 
A

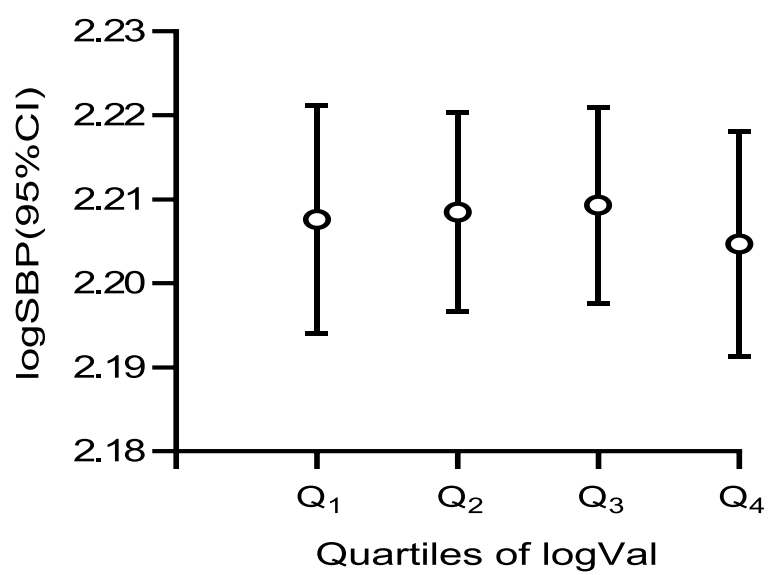

B

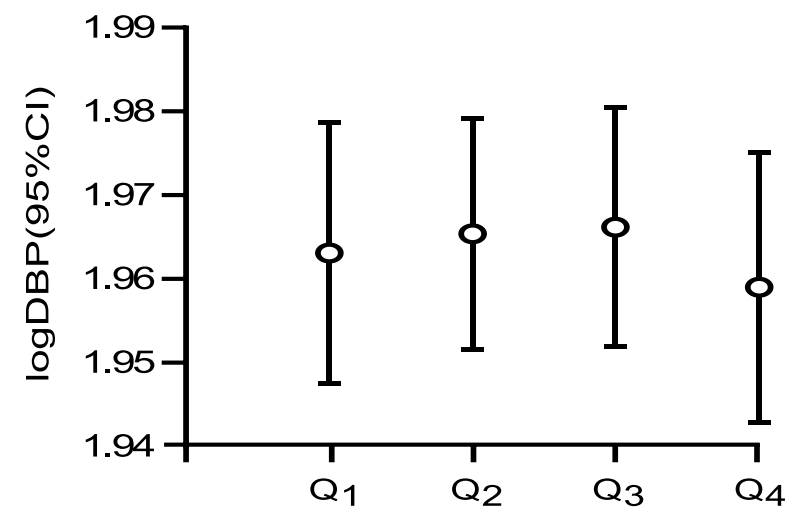

C

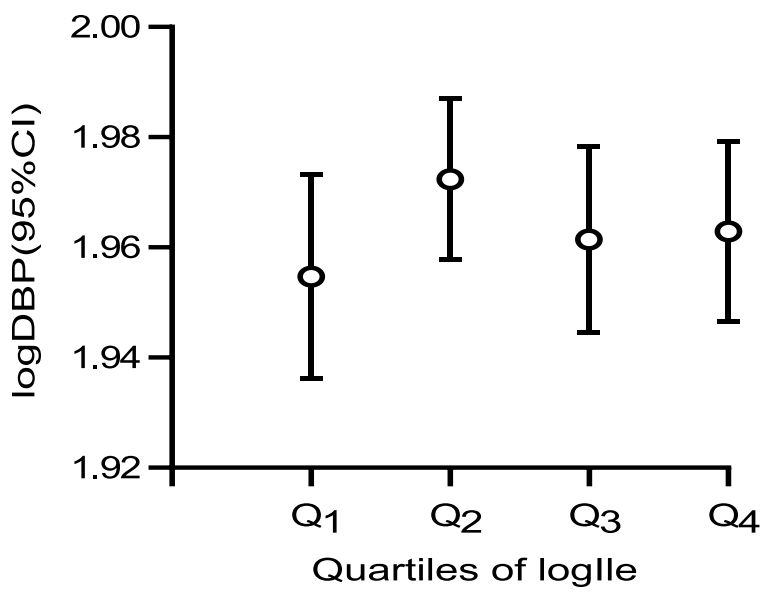

Figure I Mean $(95 \% \mathrm{Cl})$ SBP $(\mathrm{A}, \mathrm{P}$-trend $=0.784)$ and DBP $(\mathrm{B}, \mathrm{P}$-trend $=0.734$; , P-trend $=0.723$ ) by quartiles of $B C A A$ s on the basis of quartiles.

Notes: Figure I describes the differences in BP by quartiles of BCAAs on the basis of quartiles. Quartile cutoffs were as follows: Val: 0.0890 ( $25^{\text {th }}$ percentile), 0.2317 ( $50^{\text {th }}$ percentile), and 0.3927 ( $75^{\text {th }}$ percentile); lle, $-0.886 \mathrm{I},-0.6778$ and -0.4949 . BPs and BCAAs excretion rate are presented log values. The abscissa represents the quartiles of BCAAs, and ordinate represents logSBP $(95 \% \mathrm{Cl})$ or $\log \mathrm{DBP}(95 \% \mathrm{Cl})$. Abbreviations: SBP, systolic blood pressure; DBP, diastolic blood pressure; BCAAs, valine + leucine + isoleucine.

present was based on 24-hour urine samples, which were not affected by sampling time or diurnal variation, so we measured a unique panel of metabolites. However, there were also certain limitations. First, we cannot infer causality from this cross-sectional study. Moreover, although we revealed that AAAs excretion rate is not associated with BP, we cannot deny the possibility that they may influence it in some way. Second, the sample size was relatively small by epidemiologic standards, which may have limited the generalization of the discoveries. Finally, we adjusted for a series of confounders (ie, age, gender, height, weight, urine volume and drugs history), but there remained the possibility of some unknown and unmeasured confounders. Therefore, more cohorts of greater size are urgently required to validate the present findings.

\section{Conclusion}

In summary, the present study revealed that 24-hour urinary BCAAs excretion rate is closely correlated with BP in elderly hypertension patients, and these findings will provide new insights into the association between BACC metabolism and BP. Our findings highlight the need for more investigations to interpret the possible role of BCAAs in hypertension.

\section{Abbreviations}

BCAAs, branched-chain amino acids; AAAs, aromatic amino acids; SBP, systolic blood pressure; DBP, diastolic blood pressure; $\mathrm{CAD}$, coronary artery disease; Val, valine; Leu, leucine; Ile, isoleucine; ACEI, angiotensin conversion enzyme inhibitors; Tyr, tyrosine; Phe, phenylalanine; Trp, tryptophan; $\mathrm{Q}_{1}$, first quartile; $\mathrm{Q}_{2}$, second quartile; $\mathrm{Q}_{3}$, third quartile; $\mathrm{Q}_{4}$, fourth quartile; $\beta$, the standardized partial regression coefficient; $95 \% \mathrm{CI}, 95 \%$ confidence interval.

\section{Data Sharing Statement}

The datasets used and/or analysed during the current study are available from the corresponding author on reasonable request.

\section{Ethics Approval and Consent to Participate}

The Ethics Committee of Ningxia Medical University approved the present study (No. 2013-175).

\section{Acknowledgments}

We are grateful to all participants in the present study, doctors of each Rural Hospital, and the data collection staff, without them the work of the research could never have been completed. 


\section{Author Contributions}

FW and BW conducted biological specimen processing, experimental development and draft writing. WL, GW, XL, XL, NL, JZ, TY, JJ, XC and YJ participated in collecting data and biological samples needed for the investigation. $\mathrm{YZ}$ and YZ revised and improved the first draft. All authors contributed to data analysis, drafting or revising the article, have agreed on the journal to which the article will be submitted, gave final approval for the version to be published, and agreed to be accountable for all aspects of the work.

\section{Funding}

This research is a sub-study of the Salt Substitute and Stroke Study (SSaSS), which is supported by the National Health and Medical Research Council of Australia (NHMRC) Project Grant (APP1049417), NHMRC Program Grant (APP1052555); NHMRC Centre for Research Excellence Grant (APP1117300); Scientific Research Projects at the school level of Ningxia Medical University (XT2017032); and College Students Innovation Entrepreneurship Training Program (NXCX2018-135). The funders had no role in the study design, data collection, data analysis, data interpretation or writing of the report.

\section{Disclosure}

The authors declare that they have no competing interests.

\section{References}

1. Redon J, Tellez-Plaza M, Orozco-Beltran D, et al. Impact of hypertension on mortality and cardiovascular disease burden in patients with cardiovascular risk factors from a general practice setting: the ESCARVAL-risk study. $J$ Hypertens. 2016;34:1075-1083. doi:10.1097/HJH.0000000000000930

2. Wang W, Jiang B, Sun H, et al. Prevalence, incidence, and mortality of stroke in China results from a nationwide population-based survey of 480687 adults. Circulation J. 2017;135(8):759. doi:10.1161/ CIRCULATIONAHA.116.025250

3. Whelton PK, He J, Appel LJ, et al. Primary prevention of hypertension clinical and public health advisory from the national high blood pressure education program. JAMA. 2002;288(15):1882-1888. doi:10.1001/jama.288.15.1882

4. Rinschen MM, Guijas A, Palermo N. Metabolic rewiring of the hypertensive kidney. Sci Signal. 2019;12:eaax9760. doi:10.1126/scisignal.aax 9760

5. Rebholz CM, Friedman EE, Powers LJ, Arroyave WD, He J, Kelly TN. Dietary protein intake and blood pressure: a meta-analysis of randomized controlled trials. Am J Epidemiol. 2012;176(Suppl 7): S27-43. doi:10.1093/aje/kws245

6. Jennings A, MacGregor A, Welch A, Chowienczyk P, Spector T, Cassidy A. Amino acid intakes are inversely associated with arterial stiffness and central blood pressure in women. $J$ Nutr. 2015;145:2130-2138. doi:10.3945/jn.115.214700
7. Tuttle KR, Milton JE, Packard DP, Shuler LA, Short RA. Dietary amino acids and blood pressure: a cohort study of patients with cardiovascular disease. Am J Kidney Dis. 2012;59:803-809. doi:10.1053/j.ajkd.2011.12.026

8. Stamler J, Brown IJ, Daviglus ML, et al. Glutamic acid, the main dietary amino acid, and blood pressure: the INTERMAP study (international collaborative study of macronutrients, micronutrients and blood pressure). Circulation. 2009;120:221-228. doi:10.1161/ CIRCULATIONAHA.108.839241

9. Stamler J, Brown IJ, Daviglus ML, et al. Dietary glycine and blood pressure: the international study on macro/micronutrients and blood pressure. Am J Clin Nutr. 2013;98:136-145. doi:10.3945/ ajcn.112.043000

10. Dong JY, Qin LQ, Zhang Z, et al. Effect of oral L-arginine supplementation on blood pressure: a meta-analysis of randomized, double-blind, placebo-controlled trials. Am Heart $J$. 2011;162:959-965. doi:10.1016/j.ahj.2011.09.012

11. Javidan AN, Sabour H, Nazari M, et al. Is the pattern of dietary amino acids intake associated with serum lipid profile and blood pressure among individuals with spinal cord injury? J Spinal Cord Med. 2017;40:201-212. doi:10.1080/10790268.2015.1109761

12. Yang R, Dong J, Zhao H, et al. Association of branched-chain amino acids with carotid intima-media thickness and coronary artery disease risk factors. PLoS One. 2014;9:e99598. doi:10.1371/journal. pone. 0099598

13. Yamaguchi N, Mahbub MH, Takahashi H, et al. Plasma free amino acid profiles evaluate risk of metabolic syndrome, diabetes, dyslipidemia, and hypertension in a large Asian population. Environ Health Prev Med. 2017;22:35. doi:10.1186/s12199-017-0642-7

14. Jennings A, Macgregor A, Pallister T, Spector T. Associations between branched chain amino acid intake and biomarkers of adiposity and cardiometabolic health independent of genetic factors: a twin study. Int $J$ Cardiol. 2016;223:992-998. doi:10.1016/j. ijcard.2016.08.307

15. Holmes E, Loo RL, Stamler J, et al. Human metabolic phenotype diversity and its association with diet and blood pressure. Nature. 2008;453:396-400. doi:10.1038/nature06882

16. Nikolic SB, Sharman JE, Adams MJ, Edwards LM. Metabolomics in hypertension. $J$ Hypertens. 2014;32:1159-1169. doi:10.1097/ HJH.000000000000168

17. Kell DB, Brown M, Davey HM, Dunn WB, Spasic I, Oliver SG. Metabolic footprinting and systems biology: the medium is the message. Nat Rev Microbiol. 2005;3:557-565. doi:10.1038/ nrmicro1177

18. Bictash M, Ebbels TM, Chan Q, et al. Opening up the "Black Box": metabolic phenotyping and metabolome-wide association studies in epidemiology. J Clin Epidemiol. 2010;63:970-979. doi:10.1016/j. jclinepi.2009.10.001

19. Neal B, Tian M, Li N, et al. Rationale, design, and baseline characteristics of the salt substitute and stroke study (SSaSS)-A large-scale cluster randomized controlled trial. Am Heart J. 2017;188:109-117. doi:10.1016/j.ahj.2017.02.033

20. Zhang T, Chang X, Liu W, et al. Comparison of sodium, potassium, calcium, magnesium, zinc, copper and iron concentrations of elements in 24-h urine and spot urine in hypertensive patients with healthy renal function. $J$ Trace Elements Med Biol. 2017;44:104-108. doi:10.1016/j.jtemb.2017.06.006

21. Kainulainen H, Hulmi JJ, Um K. Potential role of branched-chain amino acid catabolism in regulating fat oxidation. Exerc Sport Sci Rev. 2013;41(4):194-200. doi:10.1097/JES.0b013e3182a4e6b6

22. O'Connell TM. The complex role of branched chain amino acids in diabetes and cancer. Metabolites. 2013;3:931-945. doi:10.3390/ metabo3040931

23. Sacco GD, Accinni R, Noce CD, Muratori F. C014 - Plasma amino acid pattern can be influenced by age and diabetes. Annales d'Endocrinologie. 2004;65:110-111. 
24. Guevara-Cruz M, Vargas-Morales JM, Mendez-Garcia AL, et al. Amino acid profiles of young adults differ by sex, body mass index and insulin resistance. Nutr, Metab, Cardiovasc Dis. 2017;28:393-401. doi:10.1016/j.numecd.2018.01.001

25. Katagiri R, Goto A, Budhathoki S, et al. Association between plasma concentrations of branched-chain amino acids and adipokines in Japanese adults without diabetes. Sci Rep. 2018;8:1043. doi:10.1038/s41598-018-19388-w

26. Yamakado M, Nagao K, Imaizumi A, et al. Plasma free amino acid profiles predict four-year risk of developing diabetes, metabolic syndrome, dyslipidemia, and hypertension in Japanese population. Sci Rep. 2015;5:11918. doi:10.1038/srep11918

27. Magnusson M, Lewis GD, Ericson U, et al. A diabetes-predictive amino acid score and future cardiovascular disease. Eur Heart J. 2013;34:1982-1989. doi:10.1093/eurheartj/ehs424

28. Mahbub MH, Yamaguchi N, Hase R, et al. Plasma branched-chain and aromatic amino acids in relation to hypertension. Nutrients. 2020;12:3791.

29. Tobias DK, Lawler PR, Harada PH, et al. Circulating branched-chain amino acids and incident cardiovascular disease in a prospective cohort of US women. Circulation Genomic Precision Med. 2018;11:e02157. doi:10.1161/CIRCGEN.118.002157

30. Mangge $\mathrm{H}$, Zelzer S, Pruller F, et al. Branched-chain amino acids are associated with cardiometabolic risk profiles found already in lean, overweight and obese young. J Nutr Biochem. 2016;32:123-127. doi:10.1016/j.jnutbio.2016.02.007
31. Nagata C, Nakamura K, Wada K, Tsuji M, Tamai Y, Kawachi T. Branched-chain amino acid intake and the risk of diabetes in a Japanese community: the Takayama study. Am J Epidemiol. 2013;178:1226-1232. doi:10.1093/aje/kwt112

32. Wang SM, Yang RY, Wang M, et al. Identification of serum metabolites associated with obesity and traditional risk factors for metabolic disease in Chinese adults. Nutr, Metab, Cardiovasc Dis. 2018;28:112-118. doi:10.1016/j.numecd.2017.09.009

33. De Moraes ACF, Bel-Serrat S, Manios Y, et al. Dietary protein and amino acids intake and its relationship with blood pressure in adolescents: the Helena study. Eur J Public Health. 2015;25:450-456. doi:10.1093/eurpub/cku233

34. Adachi Y, Ono N, Imaizumi A, et al. Plasma amino acid profile in severely frail elderly patients in Japan. Int $J$ Gerontol. 2018;12:290-293. doi:10.1016/j.ijge.2018.03.003

35. Farshad Teymoori GA, Parvin Mirmiran AFA. High dietary intake of aromatic amino acids increases risk of hypertension. J Am Society Hypertension. 2018;12:25-33. doi:10.1016/j.jash.2017.11.004

36. Altorf-van der Kuil W, Engberink MF, De Neve M, et al. Dietary amino acids and the risk of hypertension in a Dutch older population: the Rotterdam study. Am J Clin Nutr. 2013;97:403-410. doi:10.3945/ ajcn. 112.038737

Diabetes, Metabolic Syndrome and Obesity: Targets and Therapy

Dovepress

\section{Publish your work in this journal}

Diabetes, Metabolic Syndrome and Obesity: Targets and Therapy is an international, peer-reviewed open-access journal committed to the rapid publication of the latest laboratory and clinical findings in the fields of diabetes, metabolic syndrome and obesity research. Original research, review, case reports, hypothesis formation, expert opinion and commentaries are all considered for publication. The manuscript management system is completely online and includes a very quick and fair peer-review system, which is all easy to use. Visit http://www.dovepress.com/testimonials.php to read real quotes from published authors.

Submit your manuscript here: https://www.dovepress.com/diabetes-metabolic-syndrome-and-obesity-targets-and-therapy-journal 